\title{
Personal air sampling and biological monitoring of occupational exposure to the soil fumigant cis-1,3-dichloropropene
}

Coronel Laboratory for Occupational and Environmental Health, Department of Human Toxicology, Academic Medical Center, University of Amsterdam, PO Box 22700, $1100 \mathrm{DE}$ Amsterdam, The Netherlands E J Brouwer A J W Verplanke

Research Institute Neurosciences Amsterdam F A De Wolff

Shell Internationale Petroleum

Maatschappij BV, Health and Safety Division, Occupational Health and Toxicology, PO Box 162, 2501 AN

Den Haag, The

Netherlands

P J Boogaard

N J Van Sittert

F E Christian

Dow Europe SA, PO Box 48, 4530 AA Terneuzen, The

Netherlands

L J Bloemen

Professor H C van Hall Institute, Hereweg 99, 9721 AA Groningen, The Netherlands M Stokkentreeff

H L Hilbrands Laboratory for soil-borne pests and diseases (HLB), PO Box 323, 9400 AH

Assen, The

Netherlands

A Dijksterhuis

A Mulder

Toxicology Laboratory, Leiden University Medical Center, PO Box 9600, 2300 RC

Leiden, The

Netherlands

F A De Wolff

Correspondence to: Dr A J W Verplanke, Arbo Unie Amsterdam en omstreken, Entrada 401, 1096 EL, Amsterdam, The Netherlands tverplan@

amsterdam.arbounie.n

Accepted 29 June 2000

E J Brouwer, A J W Verplanke, P J Boogaard, L J Bloemen, N J Van Sittert, F E Christian, M Stokkentreeff, A Dijksterhuis, A Mulder, F A De Wolff

\section{Abstract}

Objectives-To assess exposure of commercial application workers to the nematocide cis-1,3-dichloropropene (cisDCP).

Methods-The study was conducted during the annual application season, August to 15 November, in the starch potato growing region in The Netherlands. 14 Application workers collected end of shift urine samples on each fumigation day $(n=119)$. The mercapturic acid metabolite N-acetyl-S-(cis-3-chloro-2-propenyl)-Lcysteine (cis-DCP-MA) in urine was used for biological monitoring of the cis-DCP uptake. Inhalatory exposure was assessed by personal air sampling during a representative sample $(n=37)$ of the fumigation days. Extensive information was collected on factors of possible relevance to the exposure and the application workers were observed for compliance with the statutory directions for use. The inhalatory exposure during all fumigation days was estimated from the relation between the personal air sampling data and the biological monitoring data. Exposure levels were correlated with the general work practice. The fumigation equipment and procedures were in accordance with the statutory directions of use, with the exception of the antidrip systems. Two antidrip systems were used: antidrip nozzles or a compressed air system.

Results-The geometric mean exposure of the application workers was $2.7 \mathrm{mg} / \mathrm{m}^{3}$ (8 hour time weighted average); range $0.1-$ $9.5 \mathrm{mg} / \mathrm{m}^{3}$. On 25 days $(21 \%)$ the exposure exceeded the Dutch occupational exposure limit (OEL) of $5 \mathrm{mg} / \mathrm{m}^{3}$. This could mainly be explained by prolonged working days of more than 8 hours. The general work practice of the application workers was rated by the observers as good or poor. No difference in exposure to cisDCP was found in the use of none, one, or two antidrip systems. Malfunctioning of the antidrip systems and lack of experience with the compressed air system were identified as possible causes for the lack of effectiveness of these antidrip systems. The use of personal protection was not always in accordance with the statutory directions of use. Dermal exposure to liquid cis-DCP was found four times during repair and maintenance, but the biologi- cal monitoring data did not suggest a significant increase in cis-DCP uptake.

Conclusions-The application of cis-DCP in the potato growing industry can be performed at exposure concentrations below the Dutch OEL of $5 \mathrm{mg} / \mathrm{m}^{3}$ if the working days are limited to 8 hours. An injector equipped with either kind of antidrip system which is in good working order, as well as the consistent use of personal protection in accordance with the statutory directions of use, may ensure exposure concentrations below the Dutch OEL.

(Occup Environ Med 2000;57:738-744)

Keywords: cis-1,3-dichloropropene; occupational exposure; soil fumigation; N-acetyl-S-(cis-3-chloro-2propenyl)-L-cysteine

In the period 1987-90 a study was performed in the Dutch flower bulb industry to develop a biological monitoring method for measuring the uptake of the soil fumigant $1,3-\mathrm{Z} / \mathrm{E}-$ dichloropropene (cis/trans-DCP). ${ }^{12}$ Brouwer et $a l^{\beta}$ reported that 8 hour time weighted average (TWA) exposures to cis/trans-DCP ranged from 1.9 to $18.9 \mathrm{mg} / \mathrm{m}^{3}$. The Dutch OEL of 5 $\mathrm{mg} / \mathrm{m}^{3}(1 \mathrm{ppm})$ was exceeded during $30 \%$ of the observed working days. The Netherlands Ministry of Social Affairs and Employment recommended measures to reduce worker's exposure during application of DCP. Based on these recommendations, adapted statutory directions ${ }^{\star}$ for use were issued by The Netherlands Ministry of Agriculture, Nature Management, and Fisheries.

The present study was designed to assess the exposure to cis-DCP of commercial application workers during the soil fumigation season and

* The statutory directions for use include instructions for fumigation with cis-DCP. The fumigation period ends on 15 November. A full face respirator with A2P3 filters (suitable for organic chlorine compounds) should be worn during all activities in which the concentration of DCP in the inhalatory air may exceed $5 \mathrm{mg} / \mathrm{m}^{3}$. Respirators should always be worn during loading, repair, and maintenance. The filters should be renewed within 1 month or after a high exposure due to a calamity. Protective clothing, PVA gloves with long shafts, and boots should be worn during all activities with potential risk for dermal exposure to liquid DCP. The gloves should be stored outside the tractor cabin. Contaminated clothing should be removed and contaminated gloves or boots washed with plenty of water. DCP has to be loaded into the tractor container by overpressure. The goose foot injector has to be equipped with antidrip nozzles or a compressed air system to prevent spillage after lifting of the injector. The injector has to be brought to the application depth (minimal $15 \mathrm{~cm}$ ) before injecting DCP and the flow to the nozzles should be interrupted at least one meter before the injector is lifted. After fumigation the soil has to be compacted or covered with plastic. 
to investigate whether the new statutory directions for use are sufficient to ensure acceptable exposure levels. The exposure data will also be used in the study of the assessment of possible effects on the liver and kidney. These results were published elsewhere. ${ }^{4}$

In the flower bulb industry as well as in the Dutch starch potato growing region DCP is used in large amounts. The present study in the starch potato growing region in the provinces of Groningen, Drente, and Overijssel, situated in the northeast of The Netherlands, was performed in cooperation with Shell, DowElanco, and the Dutch government.

Previously, the commercially available nematocides contained a mixture of cis- and trans-1,3-dichloropropene. At present, the DCP nematocides (Telone-cis and Nematrap) consist of more than $95 \%$ of the more active isomer cis-1,3-dichloropropene.

\section{Population and methods}

POPULATION

About 55 workers apply cis-DCP in The Netherlands. About 35 of them work in the Dutch starch potato growing region in the northeast of The Netherlands, of whom 23 were selected for this study. However, in this season nine application workers did not fumigate due to heavy rains and economic factors. All people gave their informed consent before their inclusion in the study. The median age of the remaining 14 application workers was 31 (range 24-51) years, the median body mass 86 (range 75-100) kg. Six application workers were non-smokers, the others smoked 10-20 cigarettes a day. Two application workers did not drink alcohol, 10 drank less than $20 \mathrm{~g} /$ day, and two 20-50 g/day. None of the application workers had a beard. A more detailed description of the study group is published elsewhere. ${ }^{4}$

FUMIGATION AND EQUIPMENT

The fumigation season started in August (week 34) and ended on 15 November 1993 (week 45). For the potato fields $851 / 10^{4} \mathrm{~m}^{2}$ cis-DCP (Telone-cis or Nematrap) was applied. A tractor mounted tank of 4001 was loaded with cis-DCP from barrels of 2001 by overpressure. The cis-DCP was injected into the soil by a goose foot injector consisting of four goose feet (each $70 \mathrm{~cm}$ wide). On the rear of each goose foot, stainless steel tubing was connected to a nozzle for dispersion of the fumigant at a median depth of $19 \mathrm{~cm}$ (range 17.5-25 cm). The soil was compacted with two power driven rollers mounted behind the goose feet.

The fields were generally fumigated lengthways. The procedure was completed by fumigation of a total of 4-6 tracks widthways at the headlands. To reduce exposure, the nozzles were lowered to the application depth before opening the tap and the tap was closed before lifting the injector at the headlands. An antidrip system was used to prevent spillage of DCP from the nozzles after lifting the injector.

DATA COLLECTION AND CHEMICAL ANALYSES

To obtain complete information on the occupational exposure of application workers, biological monitoring was performed on all fumigation days during the 1993 season. Inhalatory exposure was assessed by personal air sampling during a representative sample of these fumigation days. The biological monitoring data were correlated with the personal air sampling data to estimate the inhalatory exposure of the fumigators to cis-DCP on the days when personal air sampling was not carried out. Real time air monitoring was performed in the tractor cabin to obtain additional information on possible exposure peaks during different fumigation tasks.

On all days, during which personal air sampling was conducted, the investigators collected data about the fumigation procedures, environmental conditions, equipment, personal protection during loading, repair, and maintenance tasks, spillage of cis-DCP, and dermal exposure. The general work practice of the application workers was rated on each day by the observers as good or poor. On each fumigation day, the application workers completed a questionnaire about the duration of fumigation, use of the respirator, and possible dermal exposure.

\section{BIOLOGICAL MONITORING}

On each fumigation day, the application workers collected urine within 3 hours after the end of the fumigation period. The participants were instructed to void and discard urine before the start of application. The urine samples were collected in $500 \mathrm{ml}$ polyethene containers. Before the season a portion of urine was collected as a blank sample. These samples did not contain detectable amounts of cis-DCP.

The samples were transported to the laboratory within 24 hours, acidified by addition of 1 $\mathrm{ml} 6 \mathrm{M} \mathrm{HCl}$ per $50 \mathrm{ml}$ urine and stored at $-20^{\circ} \mathrm{C}$ until analysis. The assessment of the concentration of the cis-DCP-MA metabolite in urine was performed according to standard operating procedures of the Shell Biomedical Laboratory, Pernis, The Netherlands. Briefly, to $1.00 \mathrm{ml}$ aliquots of the acidified urine samples $30 \mathrm{ml}$ of an aqueous solution of d5-Sphenylmercapturic acid, prepared as described previously, ${ }^{5}$ was added as the internal standard. The samples were subsequently extracted with $4 \mathrm{ml}$ ethyl acetate. The organic phase was transferred to a clean tube and evaporated in a water bath at $45^{\circ} \mathrm{C}$ under a gentle stream of nitrogen. After addition of $2 \mathrm{ml}$ methylating agent (1.25 M methanolic $\mathrm{HCl}$, prepared by passing $46 \mathrm{~g}$ hydrochloric acid gas through 11 methanol) the mixture was incubated for 30 minutes at room temperature. After the reaction was completed, the methanol was evaporated in a water bath at $45^{\circ} \mathrm{C}$ under a gentle stream of nitrogen. The dry residue was dissolved in $1.0 \mathrm{ml}$ dichloromethane by ultrasonication and $1 \mu \mathrm{l}$ aliquots (splitless injection) were analysed for the methyl ester of cis-DCP-MA by gas liquid chromatography with mass spectrometric detection using a HP5890 series II gas chromatograph equipped with a HP5917A mass selective detector and a HP7673 autosampler. A $60 \mathrm{~m} \times 0.25 \mathrm{~mm}$ (internal diameter) fused silica capillary DB-1 
(Durabond) column was used with film thickness of $0.1 \mu \mathrm{m}$. The carrier gas was helium at $175 \mathrm{kPa}$ (flow of about $1 \mathrm{ml} / \mathrm{min}$ ). The PTV-purge injector $\left(250^{\circ} \mathrm{C}\right)$ was used in the purge mode and the transfer line was kept at $250^{\circ} \mathrm{C}$. The oven temperature programme was from $35^{\circ} \mathrm{C}$, kept for 1 minute, to $200^{\circ} \mathrm{C}$ at $10^{\circ} \%$ minute followed by an increase to $300^{\circ} \mathrm{C}$ at $5 \% \mathrm{~min}$. The final temperature of $300^{\circ} \mathrm{C}$ was kept for 7.5 minutes. The ionisation mode was electron impact with an ionisation potential of $70 \mathrm{eV}$ and an ion source temperature of $180^{\circ} \mathrm{C}$. For measurement, the following mass fragments were monitored: m/z 176 and 117 (fragments of cis-DCP-MA methyl ester) and $\mathrm{m} / \mathrm{z}$ 199 and 258 (fragments of d5-Sphenylmercapturic acid methyl ester). The peak heights of m/z 176 and 199 (internal standard) were used to measure the peaks at $\mathrm{m} / \mathrm{z} 117$ and 258 as qualifiers. The concentration of cis-DCP-MA was corrected for the urinary concentration of creatinine to adjust for variation in the dilution of the urine samples.

PERSONAL AIR SAMPLING

Inhalatory exposure was assessed by personal air sampling during a representative sample $(n=41)$ of the fumigation days. Personal air sampling was performed during the whole day when cis-DCP was handled, except when a respirator was worn or no exposure to cis-DCP could occur-for example, during lunch. For each application worker, personal air samples were collected by full period consecutive sampling during a maximum sampling period of about 4 hours. Personal air sampling tubes (petroleum-charcoal 100/50 tubes, SKC, Dorset, UK) were attached to the right lapel. Air was drawn through the petroleum-charcoal tubes by personal air sampling pumps (Metek, Du Pont de Nemours, Wilmington, DE, USA) at a flow rate of $180 \mathrm{ml} / \mathrm{min}$. Field blanks were included on each sampling day and from each application site. These tubes were labelled as a blank and date and location were indicated. The sampling pumps were calibrated before, during, and after each monitoring period with a petroleum-charcoal tube in line using a primary calibrator. The flow rate was adjusted to about $180 \mathrm{ml} / \mathrm{min}$ and the actual flow rate

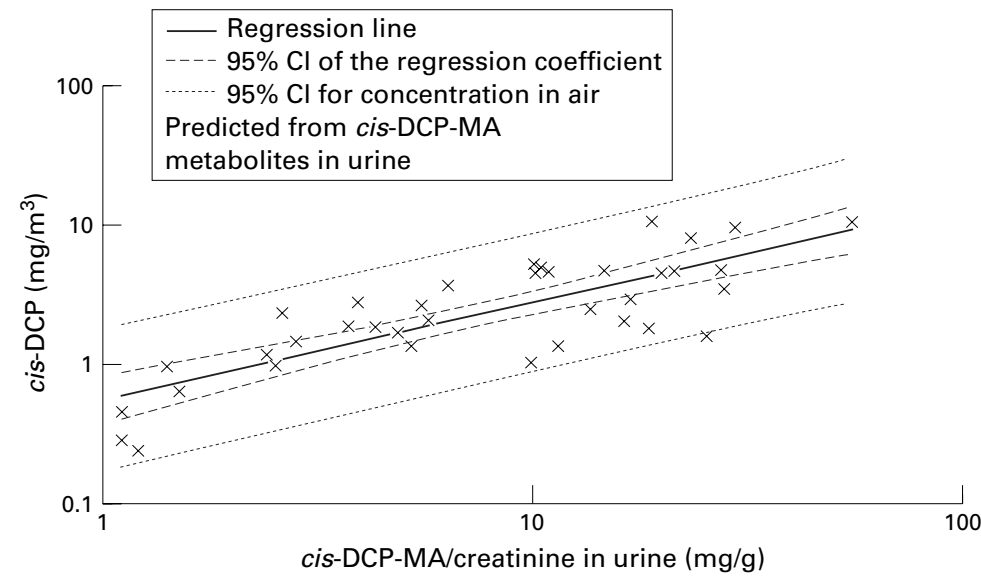

Figure 1 Correlation between personal air sampling data of cis-DCP and cis-DCP-MA (biological monitoring) in end of shift urine samples. recorded on the form provided. Starting and end times of each sampling period were noted. Immediately after sampling, the tubes were capped, labelled, and stored in a cool box at or below $0^{\circ} \mathrm{C}$. The samples were transported to the laboratory within 24 hours and stored at $-20^{\circ} \mathrm{C}$ until analysis. The DCP was analyzed according to the method described by Brouwer et al. ${ }^{3}$ In summary, cis-DCP in the personal air samples was desorbed from the charcoal by acetone. This solution was analysed by gas chromatography equipped with an electron capture detector. Cis-DCP concentrations were expressed in $\mathrm{mg} / \mathrm{m}^{3}$.

\section{CORRELATION BETWEEN BIOLOGICAL}

MONITORING AND PERSONAL AIR SAMPLING DATA Personal air sampling was performed on 41 out of 119 days; data from four days could not be used because complete personal air sampling data for the whole exposure period on those days could not be obtained. Thirty seven complete data sets were used in statistical analysis. Repeated measurement analysis did not show a significant effect of variation within people. The correlation between the 8 hour TWA air concentration of cis-DCP and the concentration of cis-DCP-MA in urine is shown in figure 1. The regression equation is:

$\log ($ cis-DCP $)=-0.255+0.709 \log$ (cis-DCPMA/urinary creatinine)

$(r=0.81$, Se $b=0.087, \mathrm{p}<0.00005)$.

The tentative biological exposure index based on these results for cis-DCP-MA is 22.2 $\mathrm{mg} / \mathrm{g}$ urinary creatinine $(95 \%$ confidence interval (95\% CI) 20.6-24.0 mg cis-DCP$\mathrm{MA} / \mathrm{g}$ urinary creatinine). These data are in good agreement with the results of Van Welie et $a l .{ }^{2}$ The correlation between the 8 hour TWA air concentration of cis-DCP and the concentration of cis-DCP-MA in urine, found by these investigators were $r=0.71$ in end of shift urine samples and $r=0.94$ in 24 hour urine samples.

REAL TIME AIR MONITORING

Real time air monitoring was carried out with a MIRAN 1B single beam infrared analyser (Foxboro, UK) in the tractor cabin during intervals of 30-120 seconds. The instrument was calibrated according to instructions of the manufacturer by injecting $0.2 \%(\mathrm{v} / \mathrm{v})$ cis-DCP in carbon disulphide into the calibration loop and analysing at a wavelength of $12.8 \mu \mathrm{m}$ with a path length of $20.25 \mathrm{~m}$.

\section{CALCULATIONS AND STATISTICS}

In the personal air sampling assessment the 8 hour TWA cis-DCP concentration was calculated for each fumigation day as:

$$
(\text { cis-DCP })=\sum_{\mathrm{i}=1}^{\mathrm{i}=\mathrm{n}}\left(\mathrm{c}_{\mathrm{i}} \times \mathrm{t}_{\mathrm{i}}\right) / 8
$$

with: $c_{i}=$ mean $c i s-\mathrm{DCP}$ concentration $(\mathrm{mg} /$ $\mathrm{m}^{3}$ ) of the sampling period $I$, and $t_{i}=$ exposure duration (h) in the sampling period $i$.

Where a total exposure period was less than 8 hours, the cis-DCP concentration during the non-exposure period was considered to be zero. 
Table 1 Exposure data from all application days $(n=119)$

\begin{tabular}{ll}
\hline Exposure time (min): & \\
AM & 521 \\
SD & 230 \\
Days $>8 \mathrm{~h}$ & $68(57 \%)$ \\
cis-DCP-MA (mg/g creatinine in urine): & 9.33 \\
GM & $0.04-55.1$ \\
Range & 2.7 \\
Calculated 8 h TWA & inhalatory exposure ( $\mathrm{mg} / \mathrm{m}^{3}$ cis-DCP): \\
GM & $0.1-9.5$ \\
Range & $25(21 \%)$ \\
Days $>$ OEL & \\
\hline
\end{tabular}

The inhalatory exposure is calculated from the relation between the PAS and the BM data (see population and methods).

The relation between inhalatory exposure to cis-DCP and urinary excretion of the mercapturic acid metabolite (cis-DCP-MA) was assessed by multiple regression analysis. Analysis of variance (ANOVA) was used to analyse the influence of work practices and other variables on the inhalatory exposure.

\section{Results}

EXPOSURE

Table 1 shows the summarised biological monitoring data from all application days, summarised estimated inhalatory exposure data and the cis-DCP-MA concentrations. The mean duration of fumigation on all $(n=119)$ application days was 521 minutes (SD 230 min) or 8.7 hours. In two cases the fumigation period was extremely long: 1140 (19 hours) and 1470 minutes (24.5 hours). On 68 days $(57 \%)$ the fumigation period exceeded 8 hours.

The geometric mean (range) excretion of cis-DCP-MA metabolites was 9.33 (0.0455.1) $\mathrm{mg} / \mathrm{g}$ urinary creatinine. The inhalatory exposure was estimated from the relation between the personal air sampling data and the biological monitoring data (see population and methods). The geometric mean (range) inhalatory exposure was $2.7(0.1-9.5) \mathrm{mg} / \mathrm{m}^{3}$ cisDCP. Comparison of the 8 hour TWA with the Dutch occupational exposure limit (OEL) of 5 $\mathrm{mg} / \mathrm{m}^{3}$ showed that the OEL was exceeded on $21 \%$ (25 days) of all application days. On 18 $(72 \%)$ out of these 25 days the fumigation period was longer than 8 hours. A cumulative frequency diagram of the estimated inhalation exposure is shown in figure 2 .

Of the days with personal air sampling and work practice observations $(n=37)$ the Dutch OEL was exceeded on 6 days $(16 \%$ of the

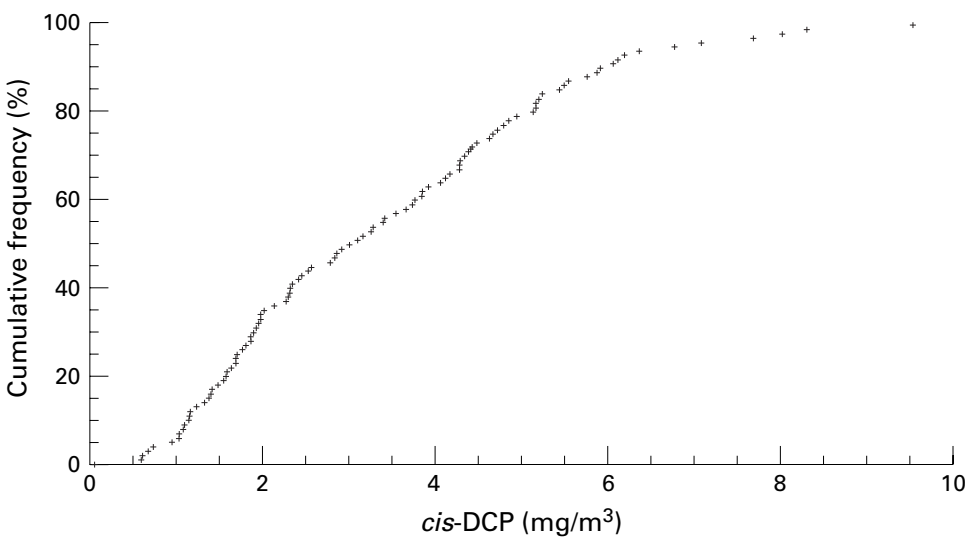

Figure 2 Cumulative frequency diagram of the estimated inhalatory exposure to cis-DCP. observed days). On 2 out of these 6 days the work practice was rated as poor, on 2 other days the cis-DCP tubing was leaking. On the remaining 2 days no specific problems were found.

Real time air monitoring was performed on 4 days: during lengthwise fumigation of the field $(n=7)$ and during turning at the headlands or fumigation of the headlands $(n=7)$. Exposure peaks during normal fumigation were less than $9 \mathrm{mg} / \mathrm{m}^{3}$ cis-DCP in five of the seven measurements. In two measurements higher peak exposures (up to $13 \mathrm{mg} / \mathrm{m}^{3}$ ) were found.

Exposure peaks during turning at the headlands or fumigation of the headlands were less than $9 \mathrm{mg} / \mathrm{m}^{3}$ cis-DCP in four measurements; in three measurements peak exposures up to $15 \mathrm{mg} / \mathrm{m}^{3}$ were found. The higher peak exposures all occurred with one goose foot injector with malfunctioning antidrip nozzles. Spillage of cis-DCP from the antidrip nozzles was found at the headlands in this case.

\section{EQUIPMENT AND PERSONAL PROTECTION}

The statutory directions of use provide instructions for the equipment, fumigation procedures, and personal protection. The compliance with these statutory directions for use was examined on the days with personal air sampling $(n=37)$. The results are summarised in figure 3. Equipment and fumigation procedures complied with the statutory directions of use with the exception of the antidrip system. On 21 days $(57 \%)$, injectors equipped with antidrip nozzles were used to prevent spillage, on 8 days $(21 \%)$ compressed air was used to clean the tubing and nozzles before lifting the injector, and on 1 day (3\%) both systems were used simultaneously. On 7 days (19\%) no system to prevent spillage was used. No difference in 8 hour TWA exposure (personal air sampling) was found with the use of the different antidrip systems (table 2).

The exposure to cis-DCP was not related to natural ventilation of the cabin (table 2 ).

The tanks on the tractors were loaded with cis-DCP 77 times in a total of 37 days. In 32 loading activities $(42 \%)$ the footwear worn was not in accordance with the statutory directions of use; three times (4\%) no gloves were worn at all, 74 times (96\%) the gloves were only wrist length. Mask and protective clothing were worn regularly. Dermal exposure did not occur during loading, nor did any of the application workers smell cis-DCP. Spillage was noticed on 19 occasions (25\%).

Repair and maintenance was performed 40 times in 37 days; on two occasions no data on personal protection were recorded. No mask was worn in $21(55 \%)$ out of the remaining 38 events. Repair activities $(n=16 ; 42 \%)$ with a high risk of dermal exposure were considered to be unblocking of nozzles or filters; flow problems; fixing leakages in the DCP tubing; and replacing a complete goose foot. No gloves with a long shaft were worn (wrist length gloves were worn on 11 occasions (29\%)); most of the time $(81 \%)$ footwear was not in accordance with the statutory directions of use. Spillage occurred in 17 out of 38 occasions (45\%). 


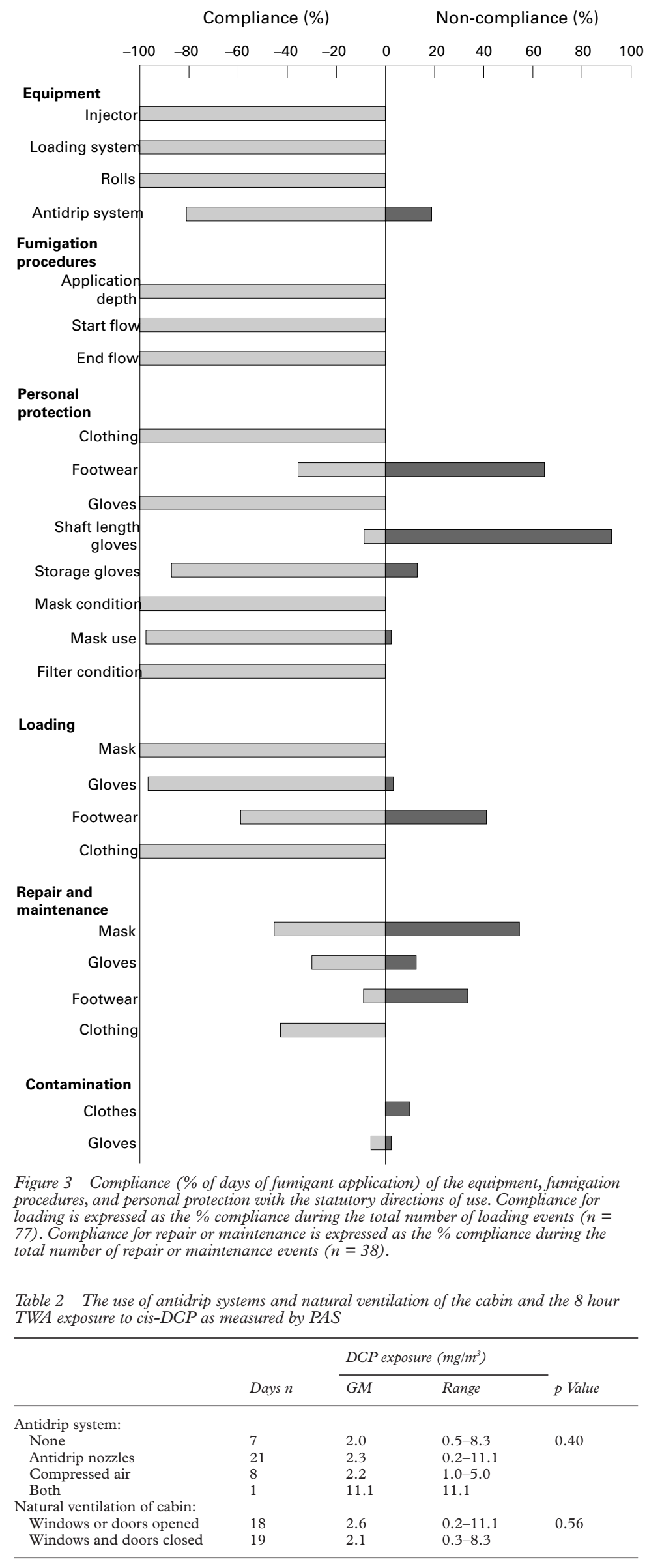

Dermal exposure occurred four times $(11 \%)$ - no gloves were worn in these casesbut the biological monitoring data did not suggest a significant increase in cis-DCP uptake. Contamination of clothes or gloves occurred seven times (18\%); twice proper measures (washing gloves) were taken.

All handling activities that were not in accordance with the statutory directions of use, $(n=171)$ were summated each day. The median number of these activities was four a day, range 1-12. The activities did not correlate with the 8 hour TWA exposure (personal air sampling) to cis-DCP $(\mathrm{p}>0.33)$.

The general work practice of the application workers was rated by the observers as poor on 4 days $(11 \%)$ and good on 33 days (89\%). Regression analysis showed a significant relation between exposure to cis-DCP and both the fumigated area and work practices. The regression equation is:

$\log$ (personal air sampling $)=-0.008+0.401$ $\mathrm{WP}+0.0364$ area.

$\left(r=0.50, \mathrm{p}=0.007\right.$, Se $\mathrm{b}_{\mathrm{WP}}=0.191$, Se $\mathrm{b}_{\mathrm{a}}$ rea $=0.014$. (personal air sampling $=8$ hour TWA exposure (personal air sampling) to cis-DCP $\left(\mathrm{mg} / \mathrm{m}^{3}\right)$, WP=dummy variable for general work practice: $0=$ good work practice; $1=$ poor work practice, area $=$ fumigated area $\left(10^{4} \mathrm{~m}^{2}\right)$.)

\section{Discussion}

Fumigation with $c i s$-DCP in the northeast of The Netherlands is performed to reduce the number of soil nematodes and prepare the fields for the next year's crop. The fumigation season is limited in time: in the period from August to 15 November fields have to be harvested, ploughed, and fumigated. Due to heavy rains in October 1993, the harvest was delayed and this restricted the number of days during which fumigation was possible. Although in agriculture it is not uncommon to work longer than 8 hours a day, the bad weather conditions contributed to prolonged working days: $68 \%$ of the fumigation days exceeded 8 hours. This should be taken into consideration when examining the results of the exposure assessment.

The OEL is based on the dose of cis-DCP absorbed in a daily 8 hour exposure period. If a worker is exposed for a shorter or longer time, the exposure is standardised to an 8 hour TWA exposure (see calculation and statistics). For instance: an exposure for 4 hours to $3 \mathrm{mg} / \mathrm{m}^{3}$ cis-DCP is standardised to an 8 hour TWA exposure of $1.5 \mathrm{mg} / \mathrm{m}^{3}$. On the other hand an exposure for 16 hours to $3 \mathrm{mg} / \mathrm{m}^{3}$ is standardised to an 8 hour TWA exposure of $6 \mathrm{mg} / \mathrm{m}^{3}$.

In this study the geometric mean (range) 8 hour TWA exposure was $2.7(0.1-9.5) \mathrm{mg} / \mathrm{m}^{3}$ cis-DCP (calculated with data from actual personal air sampling and that from estimated exposure based on biological monitoring data). The OEL was exceeded in 25 days $(21 \%)$ of all application days. On 18 of these days the application workers had worked more than 8 hours. This finding was confirmed by the correlation between the 8 hour TWA exposure and the daily fumigated area. The daily fumigated area 
is well correlated with the daily fumigation period $(r=0.98)$ : 1 hectare is fumigated in 1 hour. If the daily fumigated area is limited to 8 hectares-which is equivalent to 8 hours working - and if the general work practice is good, the estimated 8 hour TWA is 1.9 (95\% CI 1.1 to 3.2$) \mathrm{mg} / \mathrm{m}^{3}$.

The inhalatory exposure in this study was lower than in the flower bulb study in which the 8 hour TWA exposure ranged from 1.9-18.9 $\mathrm{mg} / \mathrm{m}^{3}$ but only $15 \%$ of the working days exceeded 8 hours. ${ }^{3}$ Also the peak exposures (15 $\mathrm{mg} / \mathrm{m}^{3}$ ) were considerably lower than those in the flower bulb study in which peak concentrations up to $110 \mathrm{mg} / \mathrm{m}^{3}$ were measured. ${ }^{3}$ Although the field situation and fumigation procedures in the potato growing region and the flower bulb culture are not completely comparable, the results of this study suggest that the new statutory directions of use are effective in reducing the exposure of the workers.

The OEL is defined as the exposure level that will not cause any harm or adverse effects to the health of the exposed worker or his descendants, even at daily exposures during his (occupational) life. Fumigation with cis-DCP is performed for about 3.5 months in the autumn every year. In one individual worker, the highest number of days with exposure levels of cisDCP exceeding the OEL was eight, which seems to be unimportant when compared with the conditions defined by the OEL. This is also suggested by the absence of effects on the kidney and liver, as is published elsewhere. ${ }^{4}$

Equipment and fumigation procedures complied with the statutory directions of use with the exception of the use of antidrip system. In a previous study it was found that the dripping of $c i$-DCP at the headlands caused high exposure peaks. ${ }^{3}$ Therefore, an antidrip system seemed to be necessary to prevent these exposure peaks. In the starch potato growing region the injectors were equipped with none, one, or two antidrip systems: antidrip nozzles or a compressed air system. The compressed air system, introduced in 1992, uses compressed air to clean the tubing before lifting the injector.

The measurements did not show a difference in the exposure of the application workers working with or without one or more antidrip systems. Although these systems are designed to reduce workers' exposure, the effectiveness seems to be diminished by malfunctioning. One or more antidrip nozzles were leaking on 5 days $(14 \%)$ and cis-DCP containing vapours were found after lifting the injector on 5 days $(14 \%)$. This may be partly due to lack of experience with the new compressed air system. The use of both antidrip systems simultaneously is not advisable, because the antidrip nozzles may reduce the effectiveness of the cleaning of the tubing by compressed air.

No difference was found in the maintenance of the nozzles in the different antidrip systems. Unplugging of the nozzles had to be performed on four antidrip nozzles, three compressed air system nozzles, and three nozzles when no antidrip system is used. The compressed air system introduced an unexpected type of breakdown, probably due to the high pressure used in this system: four times leakages in the tubing had to be repaired. This problem did not occur when only antidrip nozzles were used. Therefore, it is advised that the quality of the tubing of the compressed air system should be improved.

The work practice of the application workers was rated by two different methods: firstly an assessment of all activities that did not comply with the statutory directions of use, and secondly a rating by the observers of the general work practice as good or poor.

The number of tasks during which activities did not comply with the statutory directions of use did not correlate with the exposure. Two factors may explain the absence of a relation. Firstly, important preventive measures-such as the equipment and fumigation procedureswere performed mostly in accordance with the statutory directions of use and therefore, a major reduction in exposure was already achieved. Secondly, most of the activities that did not comply with the statutory directions of use are merely risks of extra exposure but are poor estimators of the actual exposure.

The general work practice, rated by the observers as good or poor, did correlate with the exposure. In rating the general work practice, the observers took into account not only the presence of preventive measures, but also the effectiveness of these measures, for instance, leaking antidrip nozzles. Thus exposures exceeding the OEL could be partly explained on at least 2 fumigation days.

The findings showed that the use of personal protection can be improved by some simple adjustments in procedures and type of protective gear. The mask was used well during loading-none of the application workers reported smelling DCP during loadinghowever, during repair or maintenance tasks the mask was not worn $55 \%$ of the time. The application workers reported two main reasons for not wearing a mask: the glass of the full face masks was misting up or the mask could not be used because the applicator wore spectacles. The first problem can be reduced by preparing the full face mask with an antimist spray; for the second problem special spectacles are available. The application workers could smell cis-DCP 11 times (29\%) during repair or maintenance. Smelling cis-DCP indicates peak exposures higher than $5 \mathrm{mg} / \mathrm{m}^{3}$. In a study with 10 volunteers, seven of them detected the odour of $5 \mathrm{mg} / \mathrm{m}^{3}{ }^{6}$ Thus, consistent use of the mask will assist in reducing exposure during repair and maintenance.

When there was need to wear gloves because of potential skin exposure, they were worn in most occasions, but their use was not consistent. During loading operations, gloves were worn on $96 \%$ of occasions, whereas during tasks involving maintenance or repair, they were worn for only $29 \%$ of the time. All but one of the application workers used wrist length gloves. In the statutory directions of use gloves with long shafts are advised, but the wrist length gloves seem to provide sufficient 
protection, because dermal exposure was only found in our study when no gloves were worn. Consistent use of gloves is advised to reduce the risk of dermal exposure during loading, repair, and maintenance tasks. Also, the gloves should be stored outside the tractor cabin.

On most days (62\%) leather safety shoes with steel toe caps were worn because these are more comfortable than rubber boots and provide better protection against mechanical risks. However, these shoes may not be adequate in protecting against dermal exposure after DCP spillage and therefore, it is advisible to change the shoes for rubber boots-preferentially with steel toe capsduring loading, repair, and maintenance tasks.

Finally, a container with a tap and filled with water should be present on the tractor, to be able to wash gloves or boots after contamination. A spare overall should be taken to the field to enable a change of protective clothing after contamination.

In general, it can be concluded that the application of cis-DCP in the starch potato growing region can be performed at exposure levels below the Dutch OEL of $5 \mathrm{mg} / \mathrm{m}^{3}$ if the working days are limited to 8 hours. An injector equipped with either kind of antidrip system, which is in good condition, as well as the con- sistent use of personal protection in accordance with the statutory directions of use may ensure exposure levels below the Dutch OEL.

We thank SA Margary (Shell International Petroleum Mij $\mathrm{BV}$ ) and $\mathrm{H}$ van der Waal (Shell Biomedical Laboratory) for their assistance in field work, J Kweekel (Shell Biomedical Laboratory) for the analyses of the urine samples, $\mathrm{H}$ van der Have (Shell Laboratory, Pernis) for the analyses of air samples, and EA van der Meulen (Erasmus University Rotterdam, Department of Epidemiology and Biostatistics) for advising on statistical analysis.

1 Welie RTH van, Duyn P van, Vermeulen NPE. Determination of two mercapturic acid metabolites of 1,3tion of two mercapturic acid metabolites of 1,3-
dichloropropene in human urine with gas chromatography dichloropropene in human urine with gas chromatography Appl 1989;496:463-71.

2 Welie RTH van, Duyn P van, Brouwer DH, et al. Inhalation exposure to 1,3-dichloropropene in the Dutch flower-bulb culture. Part II. Biological monitoring by measurement of urinary excretion of two mercapturic acid metabolites. Arch Environ Contam Toxicol 1991;20:6-19.

3 Brouwer DH, Brouwer EJ, Vreede JAF de, et al. Inhalation exposure to 1,3-dichloropropene in the dutch flower-bulb culture. Part I. Environmental monitoring. Arch Environ Contam Toxicol 1990;20:1-5.

4 Verplanke AJW, Bloemen LJ, Brouwer EJ, et al. Occupational exposure to cis-1,3-dichloropropene: biological effect monitoring of kidney and liver function. Occup Environ Med 2000;57:745-51.

5 Boogaard PJ, Sittert NJ van. Biological monitoring of exposure to benzene: a comparison between S-phenylmercapturic acid, trans,trans-muconic acid, and phenol. Occup Environ Med 1995;52:611-20

6 Albrecht WN, Chenchin K. Dissipation of 1,2-dibromo-3chloropropane (DBCP), cis-1,3-dichloropropene (1,3DCP), and dichloropropenes from soil to atmosphere. Bull Environ Contam Toxicol 1985;34:824-31. 